\title{
Effect of Liquid Crystal Material on Polymer Network Structure in Polymer Stabilized Liquid Crystal Cell
}

\author{
Rumiko Yamaguchi, Kota Inoue, and Ryo Kurosawa \\ Department of Electrical and Electronic Engineering, \\ Graduate School of Engineering and Resource Science, Akita University, \\ 1-1 Tegata gakuenmachi, Akita, 010-8502, Japan
}

\begin{abstract}
Polymer stabilized liquid crystal (PSLC) cells have been fabricated by using a reactive mesogen (RM) and various LC materials. It is clarified that a polymer network structure changes by selecting not only RM but also LC materials. Using the LC with a tolane substance, a "rice grain like" morphology can be obtained, which results in low threshold and driving voltages even if a dielectric constant anisotropy of the $\mathrm{LC}$ is very small. On the other hand, a "smooth stranded network" morphology causes the high driving voltage. When two LC materials which contribute to the smooth stranded network morphology are mixed and the LC mixture is used to fabricate the PSLC cell, the rice grain like morphology is formed. The driving voltage is lower than that in cells using two pure LCs. A relationship between solubility of the RM in the LC and the morphology formation is not observed.
\end{abstract}

Keywords: polymer stabilized liquid crystal, reverse mode, light scattering, morphology, reactive mesogen

\section{Introduction}

A nematic LC / polymer composite system has been studied as a novel electro-optical material. The most typical composite cell is a polymer dispersed liquid crystal (PDLC) [1-3] which shows a light scattering without a voltage application (off-state) and the film turns to a transparent when the voltage is applied (on-state). On the other hand, the composite cell which shows a transparent off-state and a light scattering on-state has been proposed, that is, a reverse mode.

The reverse mode cell has been proposed by Hikmet [4], which is composed of a homogeneously oriented photo polymerized LC called a reactive mesogen (RM) and the nematic LC with positive dielectric anisotropy. The RM is dissolved in the LC typically less than $10 \mathrm{wt} \%$ and is stabilized the LC molecular orientation in a bulk of the cell, which is called a polymer stabilized LC (PSLC) technology. The nematic LC with positive dielectric anisotropy allows us to select the LC with high dielectric and refractive index anisotropies among various types of LC materials, which make it possible to easily optimize display properties. The homogeneously oriented reverse mode LC film needs a sheet of polarizer which axis is parallel to the LC axis, since a polarized light perpendicular to the LC axis is not scattered by applying the voltage. To solve the highly dependence on the polarization direction of the incident light, we have proposed reverse mode LC films with $90^{\circ}$ twisted nematic (TN) $[5,6]$ and $270^{\circ}$ super twisted nematic (STN) orientation [7].

A driving voltage in the PSLC cell is generally high compared to that in a conventional LC display. It is well known that the morphology strongly affects to the driving voltage in such a polymer/LC composite cell. Therefore, relationships between electro-optical properties and morphologies have also been discussed in PSLC cells. The polymer morphology has mainly been controlled by using different chemical structures of the polymer material [8-13], as well as in PDLC cells.

In this report, we use various LC materials to change the polymer network morphology and investigate the electro-optical property in reverse mode PSLC cells. A "rice grain like" morphology $[10,11]$ which attributes to the low driving voltage is successfully controlled by selecting and mixing LC materials. 

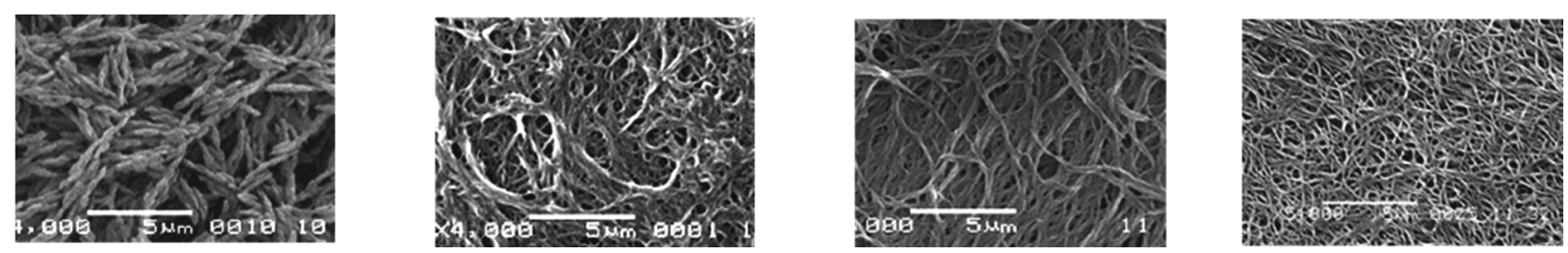

(a) MLC-2136

(b) RDP-86483

(c) E7

(d) ZLI-4792

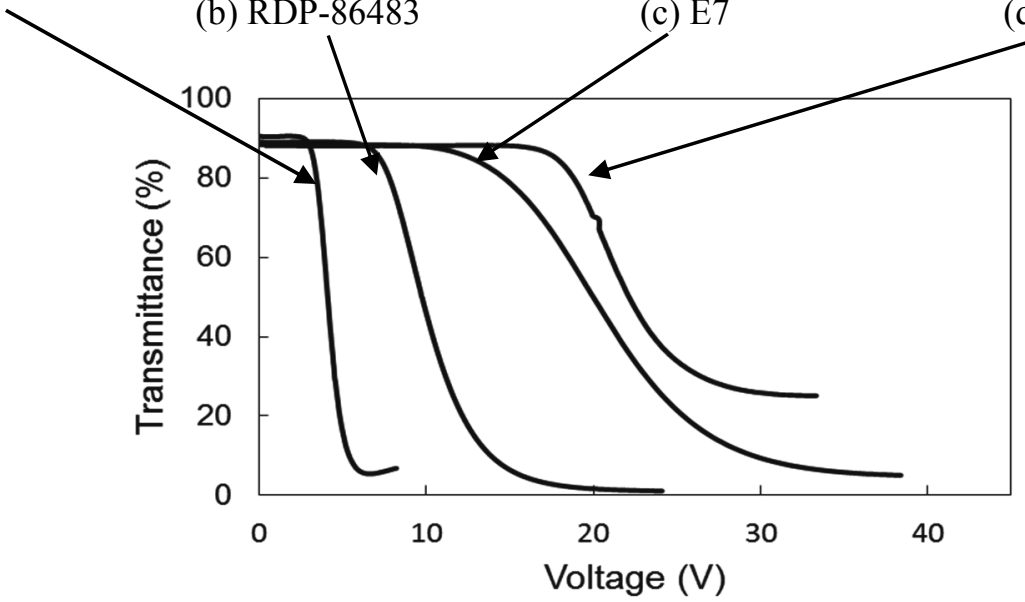

(e)

Fig. 1. (a)-(d) SEM photographs of polymer networks and (e) electro-optical properties in PSLC cells.

\section{Experimental}

The RM including a small amount of a photoinitiator and the nematic LC were mixed. The mixture was sandwiched between two glass substrates coated with rubbed polyimide (PI) film whose rubbing directions were perpendicular to each other. The gap between two substrates was 10 $\mu \mathrm{m}$. The cell was irradiated with UV light of 20 $\mathrm{mW} / \mathrm{cm}^{2}$ for 5 minutes to polymerize the $\mathrm{RM}$ keeping the twisted molecular orientation state.

The polarization plane of the incident light rotates along the molecular orientation as well as a conventional $\mathrm{TN}$ cell and the film is very clear in the off-state. Upon voltage application to the cell, LCs reorient parallel to the field and a refractive index mismatch increases between ordinary index $n_{\mathrm{o}}$ of the LC and extraordinary index $n_{\mathrm{e}}$ of the RM. Therefore, both of incident lights polarized parallel and perpendicular to the rubbing direction on an entrance side of the substrate is scattered.

Electro-optical properties were measured using a laser diode of $635 \mathrm{~nm}$ wavelength and a silicon photodiode. A frequency of the applied voltage was $1 \mathrm{kHz}$. The polarization direction of the incident light is parallel to the LC direction on the entrance side of the substrate. A collection angle of scattered light was about $2^{\circ}$. The transmittance of $100 \%$ was defined as the light intensity detected without the cell. The threshold voltage is defined as the voltage of $V_{90}$ where the transmission decreases to $90 \%$ of the voltage-off transmission.

\section{Results and Discussion}

Electro-optical curves in PSLCs are shown in Fig. 1 with SEM photographs of polymer networks. Cells were fabricated using the mixture of the LC of $95 \mathrm{wt} \%$ and the RM (ARLM002 from Osaka organic chemical industry) of $5 \mathrm{wt} \%$. Refractive index anisotropy $\Delta n$ and dielectric anisotropy $\Delta \varepsilon$ of LCs are shown in Table 1. The transmittance in the off-state is almost the same level in these PSLC cells and that in the on-state is lower with higher $\Delta n$, as well as in conventional PDLC cells.

Table 1. Nematic liquid crystals.

\begin{tabular}{ccc}
\hline LC & $\Delta n$ & $\Delta \varepsilon$ \\
\hline ZLI-4792 (Merck) & 0.09 & 5.1 \\
MLC-2136 (Merck) & 0.212 & 7.1 \\
E7 (Merck) & 0.204 & 13.8 \\
RDP-86483 (DIC) & 0.30 & 28.7 \\
\hline
\end{tabular}

The threshold voltage $V_{\text {th }}$ in a conventional homogeneous LC cell is given by

$$
V_{t h}=\pi \sqrt{\frac{k_{11}}{\varepsilon_{0} \Delta \varepsilon}}
$$

where $k_{11}$ is a splay elastic constant of the LC. A typical value of $k_{11}$ is about $10-15 \times 10^{-12} \mathrm{~N}$. 


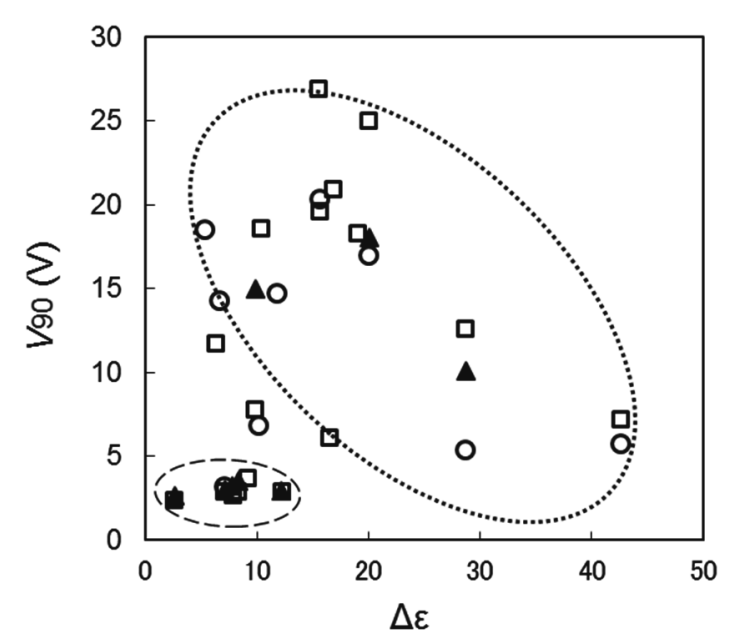

Fig. 2. Relationship between $V_{90}$ and $\Delta \varepsilon$ in PSLC cells. $\Delta$ : UCL-017 series, $\square:$ RM256 series, $\circ$ : ARLM002 series.

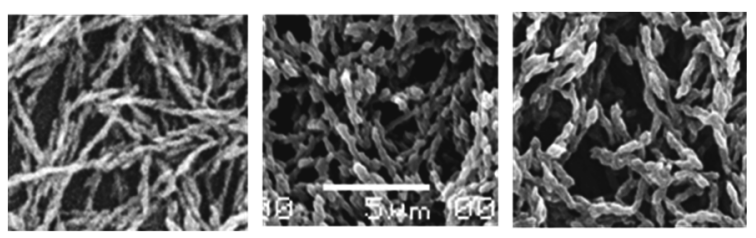

Fig. 3. SEM photographs of the rice grain like morphology in PSLCs cell using LCs with the tolane substance.

Therefore, the $V_{\text {th }}$ is generally lower by higher $\Delta \varepsilon$. However, the cell using the LC of MLC-2136 with smaller $\Delta \varepsilon$ shows the lowest $V_{\text {th }}$ in these cells. The polymer morphology shown in Fig. 1(a) is discrete "rice grain like" structure and others shown in Figs. 1(b)-(d) are "smooth stranded network" structures. Such two types of morphologies have been reported by I. Dierking et al. [10,11]. They have changed morphologies by using different RM materials. The "rice grain like" structure resulted in lower driving voltage and the "smooth stranded network" caused higher diving voltage. Almost the same results are obtained between the morphology and the driving voltage when the LC materials are selected.

More LC materials with different chemical structure and physical characteristics are investigated and the relationship between $V_{90}$ and $\Delta \varepsilon$ is shown in Fig. 2. PSLC cells were fabricated using RMs, selected from UCL-017 (DIC), RM-256 (Merck) or ARLM002. The RM concentration is $5 \mathrm{wt} \%$. These data can be roughly classified two groups. $V_{\text {th }}$ tends to follow the equation (1) in cells plotted within a dot line. Data which show very low $V_{\text {th }}$ and small $\Delta \varepsilon$ are plotted in a broken line. These low $V_{\text {th }}$ cells are fabricated using LCs with a tolane substance $(-\mathrm{C} \equiv \mathrm{C}-$ ) between phenyl rings, as well as

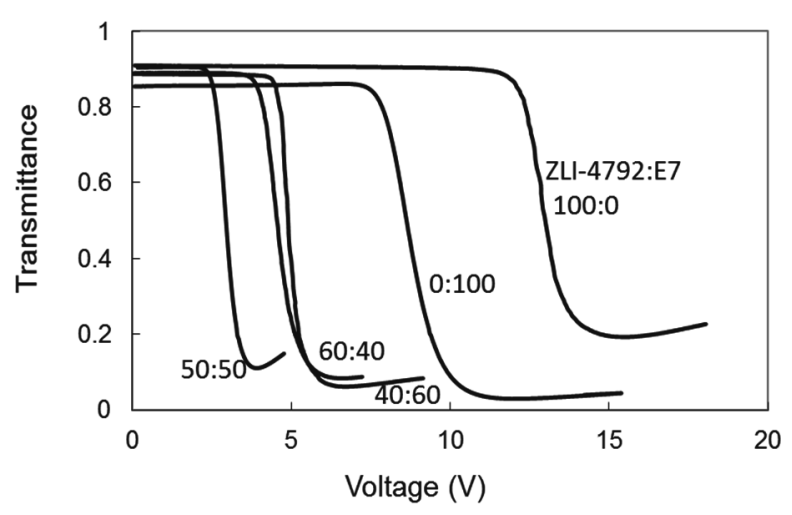

Fig. 4. Electro-optical properties in PSLC cells using mixture LCs of ZLI-4792 and E7.

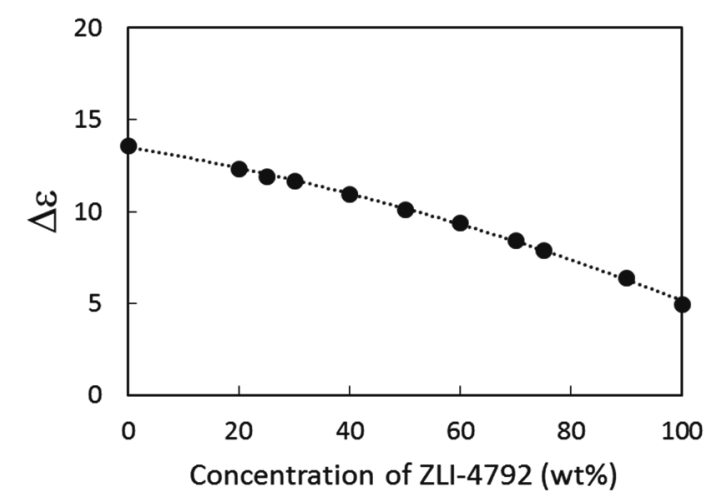

Fig. 5. $\Delta \varepsilon$ of the mixture LC of ZLI-4792 and E7.

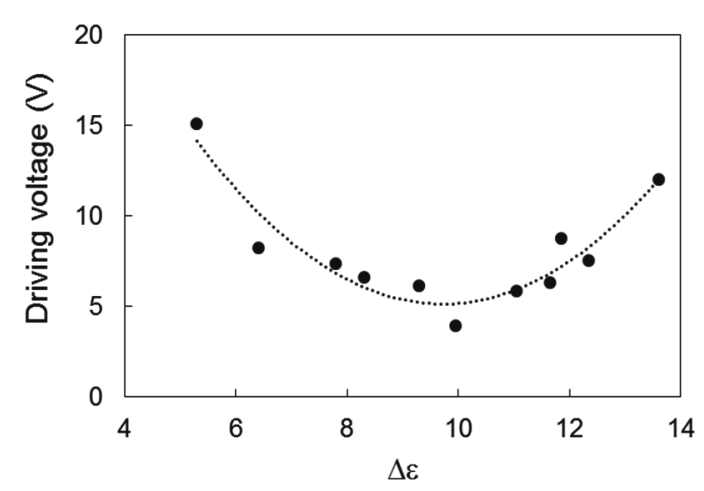

Fig. 6. Driving voltage as a function of $\Delta \varepsilon$ of LC mixture of ZLI-4792 and E7.

MLC-2136. Moreover, morphologies are the "rice grain like" type as shown in Fig. 3.

I. Dierking and Y. Yin have controlled morphologies by selecting RM materials. Dierking et al. have reported that the morphology is attributed to the solubility of the monomer within the $\mathrm{LC}$, that is, poorly soluble monomers and soluble monomers result in the "rice grain like" and the "smooth network", respectively [11]. On the other hand, Y. Yin et al. have reported that different types of morphology can be obtained even if by using poor solubility monomers [12]. In addition, our results 


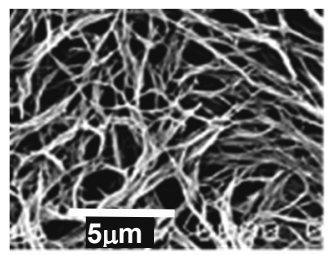

(a)

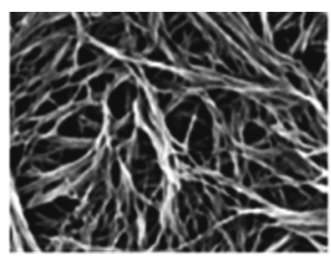

(b)

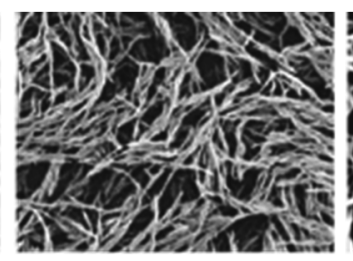

(c)

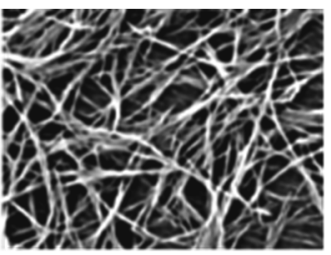

(d)

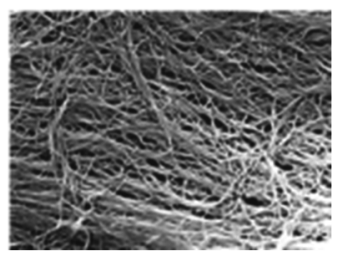

(e)

Fig. 7. SEM photographs of polymer morphologies in the PSLC cells. ZLI-4792: E7 is (a) $0: 100$, (b) $25: 75$, (c)

$50: 50$, (d) $75: 25$, and (e) $100: 0$ in weight.

show that the morphology can also be controlled by selecting LC materials.

The effect of the monomer solubility on the morphology should be discussed in the case of selected LC materials. E7 is a mixture contains cyanobiphenyl and cyanoterphenol components and the monomer of ARLM002 more than $30 \mathrm{wt} \%$ can be dissolved in E7. On the other hand, the solubility in fluorinated nematic mixture ZLI-4792 is very poor. It is difficult to dissolve the monomer more than $6 \mathrm{wt} \%$ in ZLI-4792. However, the smooth network morphology is obtained when using both E7 and ZLI-4792, as shown in Fig. 1(c) and (d). This result indicates that the solubility of the monomer is not the nature of the formation of polymer morphologies.

Next we mixed E7 and ZLI-4792, and fabricated PSLC cells. The RM used was ARLM002 of $3 \mathrm{wt} \%$. Voltage-transmittance curves of cells with various mixture ratios are shown in Fig. 4. $V_{\text {th }}$ becomes the lowest in the cell using the LC mixture of E7 of $50 \mathrm{wt} \%$ and ZLI-4792 of $50 \mathrm{wt} \%$. $\Delta \varepsilon$ of the pure ZLI-4792 is smaller than that of E7. Therefore, $\Delta \varepsilon$ of the mixture LC decreases with increasing the concentration of ZLI-4792 as shown in Fig. 5. The driving voltage which gives the lowest transmittance shown in Fig. 4 is plotted as a function of $\Delta \varepsilon$ in Fig. 6. The driving voltage decreases with increasing $\Delta \varepsilon$ of the value from 5 to 10 , which is reasonable phenomenon as well as in usual LC devices. However, it increases when $\Delta \varepsilon$ increases from 10 to 14 .

Figure 7 shows SEM photographs of PSLC cells. When pure E7 and ZLI-4792, morphologies are "smooth stranded network" and do not change by the concentration of RM of $3 \mathrm{wt} \%$ and $5 \mathrm{wt} \%$. However, the "rice grain like" morphology can be obtained in the cell fabricated using 50:50 mixture LC, as shown in Fig. 7(c). These results strongly suggest that the "rice grain like" morphology contributes to the low driving voltage of the PSLC cell. Moreover, it indicates that the solubility of the monomer in the LC does not affect to the morphology.

\section{Conclusions}

PSLC cells are fabricated using various LC materials and electro-optical properties are investigated. The morphology of the polymerized RM changes by selecting LC materials. LCs with the tolane substance causes the "rice grain like" morphology, which results in the low threshold and driving voltages. E7 and ZLI4792 which cause the "smooth stranded network" morphology and the high driving voltage are mixed. The "rice grain like" morphology and low driving voltage can be obtained by using the LC mixture. Further study to have a better understanding of the morphology formation and interaction between LC and RM is important for improving the PSLC device.

\section{References}

1. J. W. Doane, A. Golemme, J. L. West, J. B. Whitehead Jr. and B.-G. Wu, Mol. Cryst. Liq. Cryst., 165 (1988) 511.

2. P. S. Drzaic, Liq. Cryst., 3 (1988) 1543.

3. C. G. Paul Montgomery Jr. and N. A. Vaz, Appl. Opt., 26 (1987) 738.

4. R. A. Hikmet, J. Appl. Phys., 68 (1990) 4406.

5. R. Yamaguchi, Y. Waki and S. Sato, Jpn. J. Appl. Phys., 36 (1997) 2771.

6. R. Yamaguchi, Y. Waki and S. Sato, J. Photopolym. Sci. Technol., 10 (1997) 19.

7. R. Yamaguchi and L. Xiong, Jpn. J. Appl. Phys., 49 (2010) 06023-1-32.

8. Y. K. Fung, D.-K. Yang, S. Ying, L.-C. Chien, S. Zumer and J. W. Doane, Liq. Cryst., 19 (1995) 797.

9. C. V. Rajaram, S. D. Hudson and L. C. Chien, Chem. Mater, 8 (1996) 2451.

10. I. Dierking, L. L. Kosbar, A. Afzali-Ardakani, A. C. Lowe and G. A. Held, Appl. Phys. Lett., 71 (1997) 2454.

11. I. Dierking, Adv. Mater, 12 (2000) 167.

12. Y. Yin, W. Li, H. Cao, J. Guo, B. Li, S. He, C. Ouyang, M. Cao, H. Huang and H. Yang, J. Appl. Polym. Sci., 111 (2009) 1353.

13. R. Yamaguchi, K. Goto and O. Yaroshchuk, $J$. Photopolym. Sci. Technol., 25 (2012) 313. 